\title{
Predictors of Early Readmissions in Hospitalized Patients With Gastroparesis: A Nationwide Analysis
}

\author{
Ravi B Pavurala, ${ }^{1}$ Peter P Stanich, ${ }^{2}$ Somashekar G Krishna, ${ }^{2}$ Praveen Guturu, ${ }^{3}$ Alice Hinton, ${ }^{4}$ Darwin L Conwell, ${ }^{2}$ \\ and Gokulakrishnan Balasubramanian ${ }^{2 *}$
}

${ }^{1}$ Department of Internal Medicine, The Ohio State University Wexner Medical Center, Columbus, OH, USA; ${ }^{2}$ Division of Gastroenterology, Hepatology, and Nutrition, The Ohio State University Wexner Medical Center, Columbus, OH, USA; ${ }^{3}$ Division of Gastroenterology and Hepatology, University of Texas Medical Branch, Galveston, TX, USA; and ${ }^{4}$ Division of Biostatistics, College of Public Health, The Ohio State University, Columbus, OH, USA

\section{Background/Aims}

Gastroparesis is a chronic gastrointestinal disorder that frequently presents with symptoms that are difficult to manage, necessitating frequent hospitalizations. We sought to determine the predictors of early readmission due to gastroparesis based on etiology.

\section{Methods}

We identified all adults discharged with a principal diagnosis of gastroparesis after hospitalization from the 2014 Nationwide Readmission Database. We compared etiology wise (diabetes, post-surgical, and idiopathic) early readmission. Multivariate regression analyses were performed to identify significant predictors of 30-day readmission.

\section{Results}

A total of 12689 patients were identified, $30.7 \%$ diabetic, $2.6 \%$ post-surgical, and $66.7 \%$ were idiopathic. Patients with diabetic gastroparesis were more likely to be readmitted within 30 days than idiopathic (adjusted odds ratio [aOR], 0.81; 95\% confidence interval $[\mathrm{Cl}], 0.69-0.94)$ and post-surgical gastroparesis $(\mathrm{aOR}, 0.58 ; 95 \% \mathrm{Cl}, 0.34-0.98)$. Pyloroplasty was associated with less likelihood of 30-day readmission ( $a O R, 0.45 ; 95 \% \mathrm{Cl}, 0.20-0.97$ ). In addition, male gender (aOR, 1.18; 95\% Cl, 1.02-1.37), modified Elixhauser comorbidity score $\geq 3(a \mathrm{R}, 1.38 ; 95 \% \mathrm{Cl}, 1.18-1.61)$, chronic pain syndrome (aOR, 1.41; $95 \% \mathrm{Cl}, 1.11-1.78)$, younger (18-64 years) age (aOR, 1.64; 95\% Cl, 1.34-2.00), need for percutaneous endoscopic gastrostomy/jejunostomy tube (aOR, 2.06; 95\% $\mathrm{Cl}, 1.21-3.52)$, and need for total parenteral nutrition $(\mathrm{aOR}, 1.70 ; 95 \% \mathrm{Cl}, 1.24-2.35)$ were associated with increased risk of 30-day readmission.

\section{Conclusions}

One in 5 patients was readmitted with gastroparesis within 30 days. In the diabetic group, diabetes-related complications contributed to readmissions than gastroparesis. Pyloroplasty is associated with reduced early hospital readmission. Prospective studies are needed for validation of these results.

(J Neurogastroenterol Motil 2021;27:408-418)

\section{Key Words}

Diabetes; Diabetes mellitus; Gastroparesis; Patients readmission

Received: May 11, 2020 Revised: November 6, 2020 Accepted: November 17, 2020

@ This is an Open Access article distributed under the terms of the Creative Commons Attribution Non-Commercial License (http://creativecommons. org/licenses/by-nc/4.0) which permits unrestricted non-commercial use, distribution, and reproduction in any medium, provided the original work is properly cited.

${ }^{*}$ Correspondence: Gokulakrishnan Balasubramanian, MD

Division of Gastroenterology, Hepatology, and Nutrition, The Ohio State University Wexner Medical Center, 395 W. 12th Avenue, 2nd floor, Columbus, OH 43210, USA

Tel: +1-312-636-2426, Fax: +1-614-293-6255, E-mail: Gokul.bala@osumc.edu 


\section{Introduction}

Gastroparesis is a clinical condition characterized by upper gastrointestinal (GI) tract symptoms and delayed gastric emptying in the absence of mechanical obstruction. ${ }^{1}$ While several conditions can result in this clinical entity, the most common etiologies are idiopathic (36\%), diabetic (29\%), and post-surgical (13\%) in a tertiary referral setting. ${ }^{2,3}$ Treatment of gastroparesis focuses on initial restoration of the nutritional state, optimizing glycemic control, and symptom relief by the use of prokinetics. Approximately $10 \%$ of patients fail to respond to conventional medical therapy, often requiring frequent hospitalizations for symptom control and nutritional support. $^{4,5}$

Gastroparesis related hospitalizations have been on the rise in the United States (US), contributing to the increasing economic impact of hospitalizations. The burden of gastroparesis has been rising with increased emergency department visits, hospitalizations, and resultant health care costs. ${ }^{6,7}$ This translates into a significant economic burden due to impaired quality of life, reducing daily activities resulting in unemployment, loss of income, and medical disability.

The factors associated with readmissions in this population are largely unknown and have not been systematically studied. It is unknown if gastroparesis patients from one etiology have worse outcomes compared to others. Thus, we sought to evaluate rates and predictors of 30-day readmissions in patients discharged with gastroparesis as well as the impact of the etiology of gastroparesis on health care outcomes.

\section{Materials and Methods}

\section{Data Source}

We utilized the Nationwide Readmissions Database (NRD) for our study. This is sponsored by the Agency for Healthcare Research and Quality (AHRQ) as part of the Healthcare Cost and Utilization Project (HCUP). The 2014 NRD was constructed from 22 state inpatient databases, which account for $51.2 \%$ of the total US population and $49.3 \%$ of all hospitalizations. ${ }^{9}$ All of the data is de-identified and cannot be tracked to specific individual patients. The Ohio State University Data and Specimen Policy and Human Subjects Research Policy do not require Institutional Review Board approval for population-based public data set.

The NRD was queried from January to November 2014 using the International Classification of Diseases, Ninth Revision, and Clinical Modification (ICD-9-CM) diagnosis and procedure codes. Hospitalizations for acute gastroparesis were selected using ICD-9-CM diagnostic code 536.3. All procedures performed during hospitalization and patient-related co-morbidities were identified using ICD-9-CM codes as per Supplementary Table 1.

\section{Patients and Outcomes}

All patients discharged from the hospital with a principal diagnosis of gastroparesis during 2014 were selected for potential inclusion (Fig. 1). Patients with the following were excluded: (1) age less than 18 years, (2) pregnancy, (3) post-operative vomiting, (4) psychogenic vomiting, (5) rumination disorder, (6) anorexia, and (7) bulimia. When calculating 30-day readmissions, patients discharged in the month of December 2014 were excluded to allow for follow-up duration.

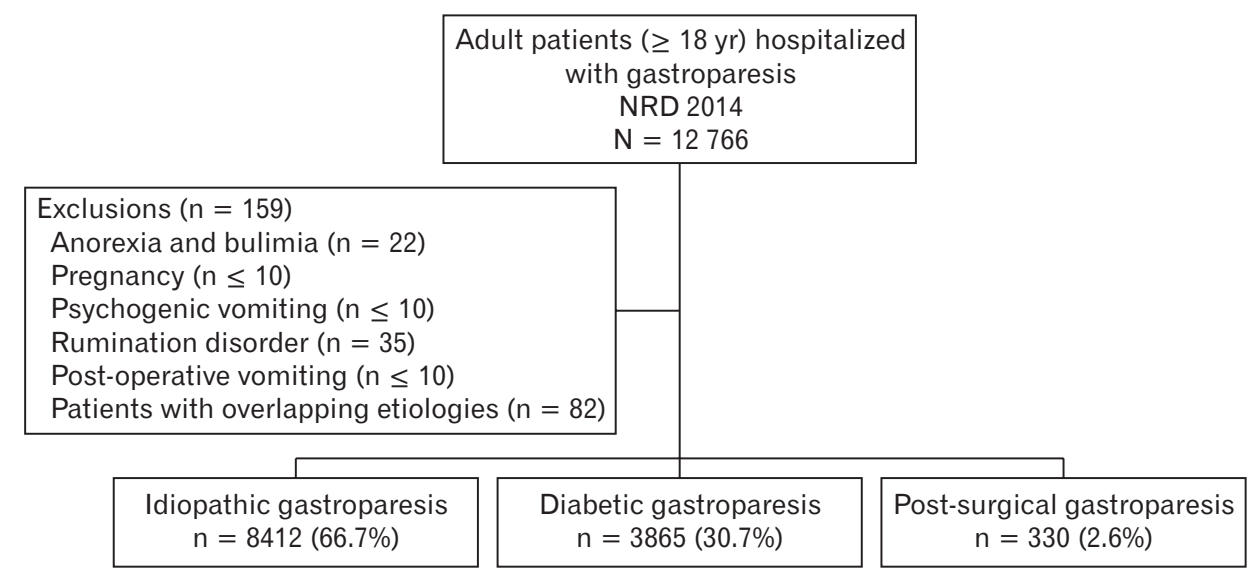

Figure 1. Study schematic and patient flow. NRD, Nationwide Readmission Database. 
Table 1. Baseline Characteristics of Patients With Gastroparesis Undergoing Hospitalization During 2014: Nationwide Readmission Database

\begin{tabular}{|c|c|}
\hline Characterisitc & $\begin{array}{l}\text { Gastroparesis } \\
(\mathrm{N}=12689)\end{array}$ \\
\hline Age (yr) & $49.82( \pm 0.31)$ \\
\hline \multicolumn{2}{|l|}{ Age group (yr) } \\
\hline $18-64$ & $10136(79.88)$ \\
\hline$\geq 65$ & $2553(20.12)$ \\
\hline \multicolumn{2}{|l|}{ Gender } \\
\hline Male & $3253(25.64)$ \\
\hline Female & $9436(74.36)$ \\
\hline \multicolumn{2}{|l|}{ Type of insurance } \\
\hline Medicare & $5206(41.10)$ \\
\hline Medicaid & $2574(20.32)$ \\
\hline Private & $3744(29.56)$ \\
\hline Other & $1142(9.01)$ \\
\hline \multicolumn{2}{|l|}{ Type of hospital } \\
\hline Urban non-teaching & $3631(28.62)$ \\
\hline Urban teaching & $8075(63.64)$ \\
\hline Rural & $983(7.74)$ \\
\hline \multicolumn{2}{|l|}{ Hospital size $^{a}$} \\
\hline Small & $1806(14.24)$ \\
\hline Medium & $3611(28.46)$ \\
\hline Large & $7272(57.31)$ \\
\hline \multicolumn{2}{|c|}{ Modified AHRQ-Elixhauser Index } \\
\hline$<3$ & $5612(44.23)$ \\
\hline$\geq 3$ & $7076(55.77)$ \\
\hline Diabetes & $3947(31.11)$ \\
\hline Cannabis/marijuana & $608(4.79)$ \\
\hline CVA & $250(1.97)$ \\
\hline Dys-autonomia & $77(0.60)$ \\
\hline Migraine & $883(6.96)$ \\
\hline Narcotics/opioid use & $585(4.61)$ \\
\hline Obesity & $1782(14.04)$ \\
\hline Scleroderma & $51(0.40)$ \\
\hline Malnutrition & $1025(8.08)$ \\
\hline Bypass for obesity & $247(1.95)$ \\
\hline Chronic pain syndrome & $880(6.94)$ \\
\hline Psychiatric disorder & $4716(37.16)$ \\
\hline Other associated GI conditions & $2781(21.92)$ \\
\hline PEG tube & $166(1.31)$ \\
\hline Percutaneous jejunostomy tube & $23(0.19)$ \\
\hline $\mathrm{TPN}$ & $493(3.89)$ \\
\hline Total gastrectomy & $11(0.09)$ \\
\hline Pyloroplasty & $171(1.35)$ \\
\hline Post-surgical gastroparesis & $412(3.25)$ \\
\hline Index admission mortality & $55(0.43)$ \\
\hline Calendar year mortality & $254(2.01)$ \\
\hline 30-Day readmission ${ }^{\mathrm{c}}$ & $3008(25.71)$ \\
\hline 90-Day readmission ${ }^{\mathrm{d}}$ & $4308(44.36)$ \\
\hline
\end{tabular}

Table 1. Continued

\begin{tabular}{lc}
\hline \multicolumn{1}{c}{ Characterisitc } & Gastroparesis \\
& $(\mathrm{N}=12689)$ \\
\hline LOS (day) & $4.96( \pm 0.08)$ \\
Cost (USD) & $9230( \pm 200)$ \\
\hline
\end{tabular}

${ }^{a}$ Hospital size categorizes are based on hospital beds and are specific to the hospital's location and teaching status. Bed size assesses the number of shortterm acute beds in a hospital. ${ }^{10}$

${ }^{\mathrm{b}}$ Comorbidities for risk adjustment were derived from Agency for Healthcare Research and Quality (AHRQ) comorbidity measures based on the methods by Elixhauser. ${ }^{13}$ Diabetes was excluded to avoid multicollinearity.

${ }^{\circ}$ December admissions and patients who died on their index admission excluded (11 699 included).

${ }^{\mathrm{d} O c t o b e r}$ November, and December admissions and patients who died on their index admission excluded (9712 included).

AHRQ, Agency for Healthcare Research and Quality; CVA, cerebrovascular accident; GI, gastrointestinal; PEG, percutaneous endoscopic gastrostomy; TPN, total parenteral nutrition; LOS, length of stay; USD, United States dollar.

Values are presented as mean $( \pm \mathrm{SE})$ or $\mathrm{n}(\%)$.

Patients with gastroparesis were further classified into $3 \mathrm{co}-$ horts; diabetic gastroparesis if patients have a history of diabetes in the same admission; post-surgical gastroparesis if patients had any of the surgeries performed during the same admission; cholecystectomy, vagotomy, Nissen fundoplication, partial gastrectomy, obesityrelated surgeries, pancreatectomy ${ }^{10,11}$; and idiopathic if patients did not have diabetes nor any of the above-mentioned surgeries. Patients with both diabetes and one of the surgeries of interest were excluded for all analyses which involved a comparison between the 3 etiologies. The primary outcome was to evaluate predictors of 30day all-cause readmissions in patients discharged with a principal diagnosis of gastroparesis after the index hospitalization. We did a subanalysis to assess the etiology of 30-day readmission in various gastroparesis cohorts using ICD-9-CM codes. Secondary outcomes were index hospitalization mortality, length of stay, and costs.

Patient-level variables included age, sex, median household income quartile for patient's zip code, and type of insurance. Comorbidities for risk adjustment were derived from AHRQ comorbidity measures based on the methods by Elixhauser et al. ${ }^{12}$ Elixhauser comorbidity scores were modified to exclude diabetes. Patients were categorized based on the number of comorbidities present: 0-2 and $\geq 3$. Other covariates included were psychiatric disorders and the presence of other GI conditions. Anxiety, bipolar disorder, depression, and schizophrenia were grouped into "psychiatric disorders." Irritable bowel syndrome, chronic constipation, slow transit constipation, dyspepsia, and other specified disorders of function of the stomach were grouped into "other associated GI conditions."

Hospital-related variables were hospital type (urban non- 
teaching, urban teaching, and rural) and hospital bed size (large, medium, and small). Hospital bed size was classified as small, medium or large based on the algorithm developed by HCUP.

\section{Statistical Methods}

The characteristics of patients discharged with gastroparesis were summarized using frequencies and percentages or means and standard errors, as appropriate. Logistic regression was used to determine the impact of etiology on and significant predictors of 30-day readmissions. Linear regression was similarly used for the outcomes of index hospitalization length of stay and cost. Terms included in the models were determined through the use of stepwise selection where age, sex, insurance type, income quartile, hospital type, hospital size, modified Elixhauser score, cannabis/marijuana, cerebrovascular accident, dysautonomia, migraine, narcotics/opioid use, obesity, scleroderma, loss of weight, malnutrition, bypass for obesity, chronic pain syndrome, psychiatric disorder, other associated GI conditions, total parenteral nutrition (TPN), percutaneous endoscopic gastrostomy (PEG)/percutaneous endoscopic jejunostomy (PEJ) tube and pyloroplasty was eligible for inclusion. Statistical significance was defined by a $P$-value 0.05 . SAS 9.4 (SAS Institute, Cary, NC, USA) using appropriate survey procedures to account for the complex study design was used to conduct all analyses and all results are weighted to represent national estimates.

\section{Results}

\section{Baseline Characteristics}

The baseline characteristics of the study cohort are shown in Table 1. Hospitalized patients with gastroparesis were predominantly women (74.4\%), less than 65 years of age, and were admitted to large urban teaching hospitals. Index admission mortality was $0.4 \%$, the mean length of stay (LOS) (standard error [SE]) was $4.96( \pm 0.08)$ days, and the mean hospital costs (SE) were 9230 ( \pm $200)$ dollars. The 30 -day readmission rate was $25.76 \%$. The mean LOS (SE) for first readmission with any cause was $5.83( \pm 0.16)$ days.

\section{Etiology Wise Outcomes of Hospitalization}

Univariate analysis (Table 2) comparing gastroparesis cohorts based on etiology revealed that patients with post-surgical gastroparesis had prolonged LOS (8.33 days) and hospitalization costs (USD 21802 ) compared to those with diabetic and idiopathic gastroparesis, albeit these outcomes include the burden of the preceding surgery. Thirty-day readmissions from any cause were higher in the diabetic gastroparesis cohort as compared to the post-surgical and idiopathic gastroparesis cohorts (29.42\% vs $17.97 \%$ vs $24.36 \%$, $P=0.001)$. On further analysis using patients readmitted within 30 days after index admission, gastroparesis specific 30-day readmission was higher in idiopathic gastroparesis compared to diabetic and post-surgical gastroparesis $(29.91 \%$ vs $11.28 \%$ vs $7.13 \%, P<$

Table 2. Comparison of Calendar Year Mortality, Index Admission Mortality, 30-Day All Cause Readmission, 30-Day Gastroparesis Specific Readmissions Among Study Population, 30-Day Gastroparesis Specific Readmissions Among Readmitted Patients, 90-Day Readmission, Length of Stay, and Index Hospitalization Costs Based on Etiology

\begin{tabular}{|c|c|c|c|c|}
\hline Outcome & $\begin{array}{c}\text { Idiopathic } \\
\text { gastroparesis } \\
(\mathrm{n}=8412)\end{array}$ & $\begin{array}{c}\text { Diabetic } \\
\text { gastroparesis } \\
(\mathrm{n}=3865)\end{array}$ & $\begin{array}{l}\text { Post-surgical } \\
\text { gastroparesis } \\
(\mathrm{n}=330)\end{array}$ & $P$-value \\
\hline Index admission mortality & $41(0.48)$ & $12(0.32)$ & $0(0.00)$ & - \\
\hline 30-Day all cause readmission ${ }^{\mathrm{a}}$ & $1887(24.36)$ & $1054(29.42)$ & $53(17.97)$ & 0.001 \\
\hline 30-Day gastroparesis specific readmission among all patients in each group ${ }^{a}$ & $564(7.29)$ & $119(3.32)$ & $\leq 10(\leq 3.03)$ & $<0.001$ \\
\hline 30-Day gastroparesis specific readmission rate among readmitted patients & $564(29.91)$ & $119(11.28)$ & $\leq 10(7.13)$ & $<0.001$ \\
\hline 90-Day readmission ${ }^{c}$ & $2707(42.13)$ & $1516(50.91)$ & $69(28.70)$ & $<0.001$ \\
\hline LOS (day) & $5.15( \pm 0.10)$ & $4.19(0.11)$ & $8.33(0.79)$ & $<0.001$ \\
\hline Cost (USD) & $9277( \pm 234)$ & $7800(214)$ & $21802(1553)$ & $<0.001$ \\
\hline
\end{tabular}

${ }^{a}$ December admissions and patients who died on their index admission excluded. Total number of patients in each cohort was used for calculating percentage.

${ }^{b}$ December admissions, patients who died on their index admission, and patients who were not readmitted within 30 days are excluded.

'October, November, and December admissions and patients who died on their index admission excluded.

LOS, length of stay; USD, United States dollar.

Values are presented as mean $\mathrm{n}(\%)$ or $( \pm \mathrm{SE})$. 


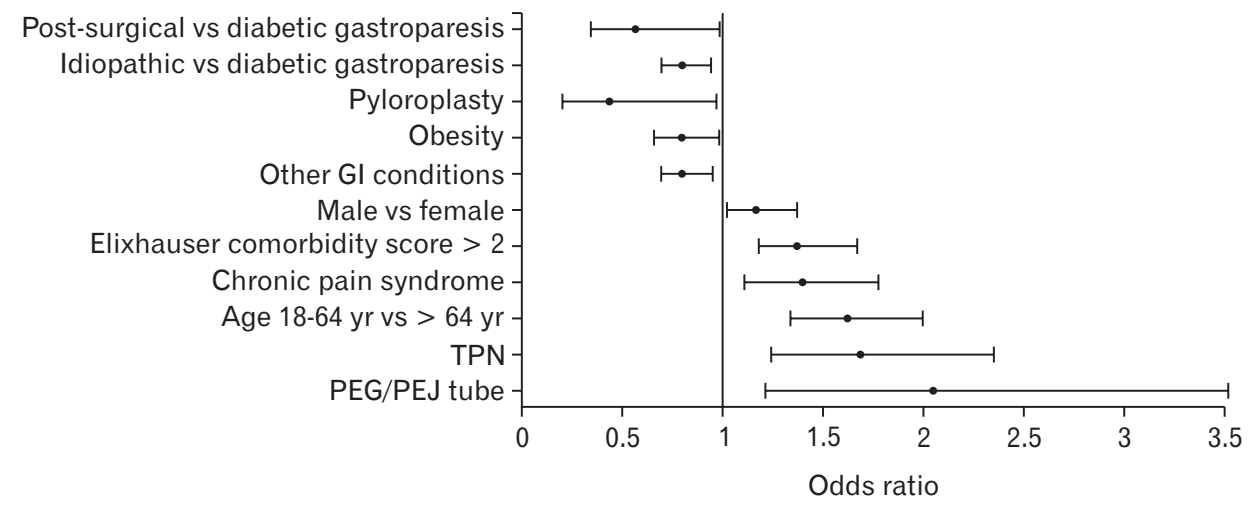

Figure 2. Predictors for early admission, multivariable analysis. GI, gastrointestinal; TPN, total parenteral nutrition; PEG, percutaneous endoscopic gastrostomy; PEJ, percutaneous endoscopic jejunostomy.

Table 3. Predictors of 30-Day Readmissions in Patients Admitted With Gastroparesis During Year 2014, Nationwide Readmission Database: Multivariate Logistic Regression Analysis

\begin{tabular}{|c|c|c|c|}
\hline \multirow{2}{*}{ Variable } & \multicolumn{3}{|c|}{ Reduced model } \\
\hline & OR & $95 \% \mathrm{CI}$ & $P$-value \\
\hline Etiology & & & 0.011 \\
\hline Diabetic gastroparesis & Reference & & \\
\hline Post-surgical gastroparesis & 0.58 & $(0.34-0.98)$ & \\
\hline Idiopathic gastroparesis & 0.81 & $(0.69-0.94)$ & \\
\hline Age group (yr) & & & $<0.001$ \\
\hline $18-64$ & 1.64 & $(1.34-2.00)$ & \\
\hline$\geq 65$ & Reference & & \\
\hline Gender & & & 0.030 \\
\hline Female & Reference & & \\
\hline Male & 1.18 & $(1.02-1.37)$ & \\
\hline Type of insurance & & & 0.002 \\
\hline Private & Reference & & \\
\hline Medicare & 1.44 & $(1.19-1.75)$ & \\
\hline Medicaid & 1.25 & $(1.03-1.52)$ & \\
\hline Other & 1.27 & $(1.00-1.63)$ & \\
\hline Type of hospital & & & 0.025 \\
\hline Rural & Reference & & \\
\hline Urban non-teaching & 1.40 & $(1.05-1.86)$ & \\
\hline Urban teaching & 1.46 & $(1.11-1.93)$ & \\
\hline Hospital size $^{a}$ & & & 0.005 \\
\hline Small & 1.27 & $(0.97-1.66)$ & \\
\hline Medium & Reference & & \\
\hline Large & 1.40 & $(1.14-1.71)$ & \\
\hline Modified Elixhauser Index $\geq 3^{b}$ & 1.38 & $(1.18-1.61)$ & $<0.001$ \\
\hline Obesity & 0.81 & $(0.66-0.98)$ & 0.033 \\
\hline Chronic pain syndrome & 1.41 & $(1.11-1.78)$ & 0.004 \\
\hline Other associated gastrointestinal conditions & 0.81 & $(0.69-0.95)$ & 0.010 \\
\hline TPN & 1.70 & $(1.24-2.35)$ & 0.001 \\
\hline PEG/PEJ tube & 2.06 & $(1.21-3.52)$ & 0.008 \\
\hline Pyloroplasty & 0.45 & $(0.20-0.97)$ & 0.042 \\
\hline
\end{tabular}

${ }^{\text {a}}$ Hospital size categorizes are based on hospital beds and are specific to the hospital's location and teaching status. Bed size assesses the number of short-term acute beds in a hospital. ${ }^{10}$

${ }^{\mathrm{b} C}$ Comorbidities for risk adjustment were derived from Agency for Healthcare Research and Quality (AHRQ) comorbidity measures based on the methods by Elixhauser. ${ }^{13}$ Diabetes was excluded to avoid multicollinearity.

TPN, total parenteral nutrition; PEG, percutaneous endoscopic gastrostomy; PEJ, percutaneous endoscopic jejunostomy. 
0.001). Less than 10 patients were readmitted with gastroparesis in the post-surgical group within 30 days. There were no deaths in the post-surgical gastroparesis cohort, while there was less than $1 \%$ mortality in the idiopathic and diabetic cohorts.

\section{Sub-group Analysis of Patients Readmitted Within 30 Days Among Various Etiological Cohorts}

Of all the patients readmitted within 30 days after index gastroparesis hospitalization, we found $22.84 \%$ were readmitted for gastroparesis and its related symptoms, whereas $14.49 \%$ were readmitted with diabetes-related complications (Supplementary Table 2A and $2 \mathrm{~B}$ ). On subgroup analysis of the diabetic gastroparesis cohort, $11.28 \%$ of patients were found to be readmitted for gastroparesis, whereas $35.36 \%$ of patients were readmitted for diabetes-related complications (Supplementary Table 3A and 3B). Among the postsurgical gastroparesis readmissions, $7.13 \%$ were from gastroparesis, $10.43 \%$ from complications secondary to organ transplant, $10.01 \%$ from complications of the surgery, and $21.87 \%$ from infectious complications (Supplementary Table 4A and 4B). In idiopathic gastroparesis readmissions, $29.91 \%$ of patients were readmitted with gastroparesis (Supplementary Table 5A and 5B). Among patients who had PEG/PEJ tube placed during index hospitalization and readmitted within 30 days, we found $16.59 \%$ of patients were from gastroparesis and related symptoms and $12.48 \%$ from gastrostomy tube complications (Supplementary Table 6).

\section{Predictors of Early Readmission}

On multivariable analysis (Fig. 2, Table 3, and Supplementary Table 7), idiopathic (aOR, 0.81; 95\% CI, 0.69-0.94) and postsurgical gastroparesis ( $\mathrm{aOR}, 0.58$; 95\% CI, 0.34-0.98) cohorts were less likely to be readmitted within 30 days compared to the diabetic gastroparesis cohort.

Similarly, obesity (aOR, 0.81; 95\% CI, 0.66-0.98), other associated GI conditions (aOR, 0.81; 95\% CI, 0.69-0.95), and pyloroplasty (aOR, 0.45 ; 95\% CI, 0.20-0.97) were associated with decreased odds of 30-day readmissions whereas, age 18-64 years (aOR, 1.64; 95\% CI, 1.34-2.00), male sex (aOR, 1.18; 95\% CI, 1.02-1.37), modified Elixhauser score $\geq 3$ (aOR, 1.38; 95\% CI, 1.18-1.61), chronic pain syndrome (aOR, 1.41; 95\% CI, 1.111.78), TPN (aOR 1.70; 95\% CI, 1.24-2.35), and percutaneous endoscopic gastrostomy/percutaneous endoscopic jejunostomy (PEG/PEJ) tube placement (aOR, 2.06; 95\% CI, 1.21-3.52) were associated with increased odds of 30 -day readmissions.

As compared to private insurance, Medicare (aOR, 1.44; 95\% CI, 1.19-1.75) and Medicaid (aOR, 1.25; 95\% CI, 1.03-1.52) were associated with higher odds of 30-day readmission. Further, patients discharged from urban non-teaching (aOR, 1.40; 95\% CI, 1.05-1.86) and urban teaching (aOR, 1.46; 95\% CI, 1.11-1.93), as well as patients discharged from large hospitals (aOR, 1.40; 95\% CI, 1.14-1.71) were associated with higher odds of 30-day readmission as compared to patients discharged from rural and medium sized hospitals respectively.

\section{Etiology Based Predictors Associated With Increased Healthcare Utilization (Length of Stay and Hospitalization Costs)}

Idiopathic gastroparesis cohort has prolonged hospital LOS (0.62 days; 95\% CI, 0.42-0.83) and increased hospital costs (US $\$ 645$; 95\% CI, 280-1009) compared to the diabetic gastroparesis cohort (Tables 4 and 5; Supplementary Tables 8 and 9). Similarly, the post-surgical gastroparesis cohort has higher healthcare utilization (LOS, 2.97 days; 95\% CI, 1.82-4.12 and hospital costs US\$11 151; 95\% CI, 9373-12 929) than the diabetes cohort.

Multiple other factors, including Modified Elixhauser score $\geq 3$, need for total parenteral nutrition, and need for PEG/PEJ tubes were associated with increased health care utilization as listed in Tables 4 and 5. On the contrary, the use of cannabis/marijuana was associated with decreased healthcare utilization (Tables 4 and 5).

\section{Discussion}

In this national database looking at 30-day readmissions among patients discharged after index hospitalization with gastroparesis, we found 1 in 4 patients were readmitted with any cause whereas 1 in 5 patients were readmitted with gastroparesis. In addition, cumulative comorbidities, younger age, male gender, chronic pain syndrome history, and need for an alternate form of nutrition were independently associated with higher 30-day readmission in patients admitted with gastroparesis, whereas pyloroplasty and the presence of other GI conditions were associated with decreased odds of 30-day readmissions. To our knowledge, this is the first study to evaluate predictors of 30-day readmissions in patients admitted with gastroparesis based on etiology in a large population database.

In our study, we found idiopathic and post-surgical gastroparesis patients are less likely to be readmitted compared to diabetic gastroparesis within 30 days of the index hospitalization. However, diabetes-related complications $(35.36 \%)$ accounted for the majority of the readmissions while only a small fraction (11.28\%) accounting for gastroparesis related readmission in diabetics. Gastroparesis is a well-known complication of diabetes mellitus ${ }^{13}$ and poor glycemic 
Table 4. Factors Associated With Length of Stay in Patients Admitted With Gastroparesis Nationwide Readmission Database 2014: Linear Regression

\begin{tabular}{|c|c|c|c|}
\hline \multirow{2}{*}{ Variable } & \multicolumn{3}{|c|}{ Reduced model } \\
\hline & Coefficient in days & $95 \% \mathrm{CI}$ & $P$-value \\
\hline Etiology & & & $<0.001$ \\
\hline Diabetic gastroparesis & Reference & & \\
\hline Post-surgical gastroparesis & 2.97 & $(1.82-4.12)$ & \\
\hline Idiopathic gastroparesis & 0.62 & $(0.42-0.83)$ & \\
\hline Age group (yr) & & & 0.001 \\
\hline $18-64$ & Reference & & \\
\hline$\geq 65$ & 0.56 & $(0.22-0.91)$ & \\
\hline Type of hospital & & & $<0.001$ \\
\hline Rural & Reference & & \\
\hline Urban non-teaching & 0.12 & $(-0.06-0.30)$ & \\
\hline Urban teaching & 0.53 & $(0.32-0.75)$ & \\
\hline Hospital size $^{a}$ & & & $<0.001$ \\
\hline Small & -0.09 & $(-0.37-0.18)$ & \\
\hline Medium & Reference & & \\
\hline Large & 0.80 & $(0.57-1.02)$ & \\
\hline Modified Elixhauser Index $\geq 3^{b}$ & 1.06 & $(0.88-1.25)$ & $<0.001$ \\
\hline Cannabis/marijuana & -1.41 & $(-1.70--1.11)$ & $<0.001$ \\
\hline CVA & 1.10 & $(0.19-2.00)$ & 0.018 \\
\hline Dys-autonomia & 1.98 & $(0.22-3.75)$ & 0.027 \\
\hline Migraine & 0.87 & $(0.36-1.37)$ & 0.001 \\
\hline Narcotics/opioid use & 0.75 & $(0.21-1.28)$ & 0.006 \\
\hline Malnutrition & 3.70 & $(3.02-4.37)$ & $<0.001$ \\
\hline Other associated gastrointestinal conditions & 0.83 & $(0.58-1.08)$ & $<0.001$ \\
\hline TPN & 6.08 & $(5.08-7.08)$ & $<0.001$ \\
\hline PEG/PEJ tube & 7.89 & $(5.61-10.16)$ & $<0.001$ \\
\hline
\end{tabular}

${ }^{a}$ Hospital size categorizes are based on hospital beds and are specific to the hospital's location and teaching status. Bed size assesses the number of short-term acute beds in a hospital. ${ }^{10}$

${ }^{\mathrm{b}}$ Comorbidities for risk adjustment were derived from Agency for Healthcare Research and Quality (AHRQ) comorbidity measures based on the methods by Elixhauser. ${ }^{13}$ Diabetes was excluded to avoid multicollinearity.

CVA, cerebrovascular accident; TPN, total parenteral nutrition; PEG, percutaneous endoscopic gastrostomy; PEJ, percutaneous endoscopic jejunostomy.

control is associated with precipitation of gastroparesis symptoms and consequent hospitalizations. ${ }^{14}$ Further, diabetic complications including autonomic neuropathy and nephropathy can worsen gastroparesis; tight glycemic control improves gastric emptying and gastroparesis symptoms ${ }^{2,15-17}$ by slowing down microvascular complications. Furthermore, diabetic patients frequently have associated comorbidities like cardiovascular disease, hypertension, and retinopathy, and metabolic syndrome which may contribute to frequent readmissions. ${ }^{18}$ Diabetic gastroparesis has a higher number of hospitalizations than idiopathic gastroparesis in the National Institute of Diabetes and Digestive and Kidney Disease Gastroparesis Research Consortium. ${ }^{19}$ Although we were unable to evaluate glycemic status using NRD, we suspect better glucose management in diabetic gastroparesis may potentially reduce rates of early read- mission by improving the gastroparesis as well as decreasing the complications related to diabetes.

Similar to the diabetic cohort, only $7.13 \%$ of readmitted patients in post-surgical gastroparesis were due to gastroparesis while catheter-related infections $(21.87 \%)$ is the major contributor for its readmissions. Patients with Nissen fundoplication appear to be commonly associated with post-surgical gastroparesis and more than $90 \%$ of these patients had resolution of symptoms by 1 year. ${ }^{20}$ Unlike the diabetic and post-surgical group, about $29.91 \%$ of readmitted patients with idiopathic gastroparesis were from gastroparesis. Though the all-cause 30-day readmissions were lower than diabetic gastroparesis, gastroparesis specific 30-day readmission was higher in patients with idiopathic gastroparesis. Idiopathic gastroparesis is a heterogeneous group of patients with various etiologies. 
Table 5. Factors Associated With Index Hospitalization Costs in Patients Admitted With Gastroparesis Nationwide Readmission Database 2014: Linear Regression Analysis

\begin{tabular}{|c|c|c|c|}
\hline \multirow{2}{*}{ Variable } & \multicolumn{3}{|c|}{ Reduced model } \\
\hline & Coefficient in dollars & $95 \% \mathrm{CI}$ & $P$-value \\
\hline Etiology & & & $<0.001$ \\
\hline Diabetic gastroparesis & Reference & & \\
\hline Post-surgical gastroparesis & 11151 & $(9373-12929)$ & \\
\hline Idiopathic gastroparesis & 645 & $(280-1009)$ & \\
\hline Income quartile & & & $<0.001$ \\
\hline First & Reference & & \\
\hline Second & 40 & $(-436-516)$ & \\
\hline Third & 791 & $(211-1370)$ & \\
\hline Fourth & 1933 & $(1239-2626)$ & \\
\hline Type of hospital & & & $<0.001$ \\
\hline Rural & Reference & & \\
\hline Urban non-teaching & -770 & $(-1235--305)$ & \\
\hline Urban teaching & 95 & $(-424-614)$ & \\
\hline Hospital size $^{a}$ & & & $<0.001$ \\
\hline Small & 218 & $(-471-906)$ & \\
\hline Medium & Reference & & \\
\hline Large & 995 & $(521-1470)$ & \\
\hline Modified Elixhauser Index $\geq 3^{\mathrm{b}}$ & 1958 & $(1462-2455)$ & $<0.001$ \\
\hline Cannabis/marijuana & -2538 & $(-3013--2062)$ & $<0.001$ \\
\hline CVA & 2721 & $(1637-3806)$ & $<0.001$ \\
\hline Dys-autonomia & 5005 & $(1439-8572)$ & 0.006 \\
\hline Migraine & 1437 & $(367-2506)$ & 0.009 \\
\hline Obesity & -808 & $(-1274--342)$ & 0.001 \\
\hline Malnutrition & 5723 & $(4297-7150)$ & $<0.001$ \\
\hline Other associated gastrointestinal conditions & 877 & $(370-1385)$ & 0.001 \\
\hline $\mathrm{TPN}$ & 12803 & $(10772-14835)$ & $<0.001$ \\
\hline PEG/PEJ tube & 12526 & $(8168-16885)$ & $<0.001$ \\
\hline Pyloroplasty & 3650 & $(2181-5119)$ & $<0.001$ \\
\hline
\end{tabular}

${ }^{2}$ Hospital size categorizes are based on hospital beds and are specific to the hospital's location and teaching status. Bed size assesses the number of short-term acute beds in a hospital. ${ }^{10}$

${ }^{\mathrm{b}}$ Comorbidities for risk adjustment were derived from Agency for Healthcare Research and Quality (AHRQ) comorbidity measures based on the methods by Elixhauser. ${ }^{13}$ Diabetes was excluded to avoid multicollinearity.

CVA, cerebrovascular accident; TPN, total parenteral nutrition; PEG, percutaneous endoscopic gastrostomy; PEJ, percutaneous endoscopic jejunostomy.

The exact cause of the higher gastroparesis specific readmissions among the idiopathic gastroparesis group is largely unknown and warrants further prospective studies.

In our study, about $7 \%$ of gastroparesis patients had chronic pain syndrome and this is associated with increased 30-day readmissions. Abdominal pain frequently was a debilitating symptom in gastroparesis ${ }^{21}$ as well as in chronic pain syndrome. The use of opioid analgesics for chronic pain syndrome could further delay gastric emptying and exacerbate gastroparesis symptoms. This may lead to readmissions and thus should be avoided. Low dose tricyclic antidepressants ${ }^{20}$ and other classes of medications including selective serotonergic reuptake inhibitors, selective norepinephrine reuptake inhibitors, gabapentin, pregabalin have been used for pain alleviation with limited benefit. ${ }^{2,22-25}$ Future research should focus on better pain management strategies to help with both improving the quality of patient's life and potentially reducing hospitalizations.

PEG/PEJ tube is offered to patients who fail to thrive due to severe symptomatic gastroparesis to improve nutritional status. While PEG tube vents the gastric contents and improves gastroparesis symptoms, $\mathrm{PEJ}$ provides access for enteral nutrition in patients with severe gastroparesis. Venting gastrostomy tube for refractory idiopathic gastroparesis is effective in relieving gastric stasis symp- 
toms and improved patients' quality of life. ${ }^{26}$ Though surgically placed venting gastrostomy reduced hospitalizations from 0.5 admissions per year to 0.1 admissions per year (a factor of 5) in colonic intestinal pseudo-obstruction, ${ }^{2728}$ studies addressing the same outcome in gastroparesis patients were lacking. In our study, we found PEG/PEJ tube has been associated with increased 30-day readmission, prolonged length of stay, and higher index hospitalization costs. On the subgroup analysis of patients with PEG/PEG-J tube, we found $16.59 \%$ of patients were readmitted with gastroparesis and related symptoms whereas $12.48 \%$ were readmitted with complications from gastrostomy tube placement. Nationwide inpatient readmission database used in this study is limited by the lack of indication for procedures like PEG tube or PEJ tube placement. We suspect the severity of gastroparesis among these patients and complications related to tube placement likely contribute to higher readmissions in this group, whereas prolonged LOS and higher index hospitalization costs could be associated with PEG/PEJ tube procedure itself.

Similarly, we noted the use of TPN was associated with an increased risk of 30-day readmissions in our study. TPN is generally used in patients with severe and uncontrolled gastroparesis leading to malnourishment. Infections, including vascular line infections, can contribute to gastroparesis exacerbation and, thus hospitalizations. ${ }^{14}$ Further, TPN is associated with complications such as catheter-related bacterial bloodstream infections, sepsis, thrombosis, and liver disease that may contribute to readmissions. ${ }^{29-31}$ Hence risks and benefits should be weighed while considering TPN because of the associated complications.

Laparoscopic pyloroplasty has improved gastroparesis cardinal symptom index scores and gastric emptying with even normalization in some patients. ${ }^{32-34}$ This was reflected in our study as it was associated with decreased odds of 30-day readmission. This management option could be offered in patients with refractory symptoms and poor quality of life. ${ }^{35}$

Cannabis use was associated with decreased length of stay and costs in our study. We did not find an association of cannabis use with 30-day readmissions. A recent retrospective study showed cannabis use had been associated with reduced gastroparesis symptoms. ${ }^{36}$ Although cannabis had been shown to delay gastric emptying, it helps reduce nausea by its central effect in patients with severe gastroparesis. ${ }^{37}$ Further randomized prospective trials are needed to validate the role of cannabis in gastroparesis.

While few studies were looking at risk factors for 30-day readmission in gastroparesis patients using NRD, our study is unique in comparing 30-day all-cause and gastroparesis specific readmissions among the 3 main etiologies of gastroparesis. In a study by Shahsavari et al, ${ }^{38}$ patients with diagnoses of both primary and secondary gastroparesis were included; marijuana use was associated with increased 30-day readmissions and diabetes with decreased 30-day readmissions. Conversely, the 30-day readmissions were not associated with marijuana use and increased in the diabetic gastroparesis group in our study. In a study by Qayed and Muftah, ${ }^{39}$ using NRD 2010-2014, looking at care fragmentation in gastroparesis patients, there was no association of PEG/PEJ tube with 30-day readmissions whereas our study found an increased association. The difference in the results in our study could be related to stringent exclusion criteria such as post-operative vomiting, eating disorder, rumination syndrome, and pregnancy-related vomiting which could mimic gastroparesis.

Our study has several limitations inherent to an administrative database dependent on ICD-9-CM coding. We utilized NRD to account for individual patient readmission rates. However, the database is limited by a lack of information on medication administration, laboratory values, and outpatient follow-up. Since the NRD is not all-inclusive, the possibility of unobserved confounding is nevertheless present. Also, we were unable to differentiate between diabetes types as there was a common ICD-9 code. However, a major advantage of utilizing the NRD is that we can study many individual/unique patients and follow them longitudinally over 1 calendar year. Since NRD is a nationally representative sample, the results are thus generalizable to the US population. LOS and hospitalization costs associated with gastroparesis in the post-surgical group include burden from the surgery itself, which can be confounding and makes it difficult to conclude. Idiopathic gastroparesis patients, categorized by exclusion, may have unaccounted variables contributing to the results. Cannabis usage could be inaccurate in our study as the coding for cannabis is questionable at best and underreporting its use. There is a possibility that patients with idiopathic gastroparesis could get one of the surgeries of interest and be categorized into post-surgical gastroparesis cohort. Similarly, some of the post-surgical patients may have been classified into idiopathic if surgery was done before January 2014 and were not captured in our database. Similarly, patients categorized to idiopathic gastroparesis group could develop diabetes later. We were unable to assess the correlation between the severity of diabetes and gastroparesis readmissions as NRD does not have information on glycosylated hemoglobin.

In conclusion, we found out 1 in 5 gastroparesis patients are readmitted with gastroparesis symptoms within 30 days. Diabetic gastroparesis patients were frequently readmitted compared to other 
etiologies, but the majority was contributed by diabetes-related complications rather than gastroparesis. Cannabis use is not associated with higher 30-day readmissions. Pyloroplasty was associated with reduced 30-day readmissions, and prospective randomized controlled studies are needed to confirm our findings and further evaluate pyloroplasty in gastroparesis management.

\section{Supplementary Materials}

Note: To access the supplementary tables mentioned in this article, visit the online version of Journal of Neurogastroenterology and Motility at http://www.jnmjournal.org/, and at https://doi. org/10.5056/jnm20105.

\section{Financial support: None.}

\section{Conflicts of interest: None.}

Author contributions: Ravi B Pavurala and Gokulakrishnan Balasubramanian: study concept and design, acquisition of data, analysis and interpretation of data, drafting of the manuscript, critical revision of the manuscript for important intellectual content, and study supervision; Peter P Stanich: study concept and design, acquisition of data, analysis and interpretation of data, drafting of the manuscript, and critical revision of the manuscript for important intellectual content; Somashekar G Krishna and Praveen Guturu: critical revision of the manuscript for important intellectual content; Alice Hinton: statistical analysis, acquisition of data, analysis and interpretation of data, and critical revision of the manuscript for important intellectual content; and Darwin L Conwell: critical revision of the manuscript for important intellectual content.

\section{References}

1. Parkman HP, Hasler WL, Fisher RS, American Gastroenterological A. American gastroenterological association medical position statement: diagnosis and treatment of gastroparesis. Gastroenterology 2004;127:15891591.

2. Camilleri M, Parkman HP, Shafi MA, Abell TL, Gerson L. Clinical guideline: management of gastroparesis. Am J Gastroenterol 2013;108:18-37.

3. Soykan I, Sivri B, Sarosiek I, Kiernan B, McCallum RW. Demography, clinical characteristics, psychological and abuse profiles, treatment, and long-term follow-up of patients with gastroparesis. Dig Dis Sci 1998;43:2398-2404.

4. Cutts TF, Luo J, Starkebaum W, Rashed H, Abell TL. Is gastric electrical stimulation superior to standard pharmacologic therapy in improving GI symptoms, healthcare resources, and long-term health care benefits?
Neurogastroenterol Motil 2005;17:35-43.

5. Nusrat S, Bielefeldt K. Gastroparesis on the rise: incidence vs awareness? Neurogastroenterol Motil 2013;25:16-22.

6. Hirsch W, Nee J, Ballou S, et al. Emergency department burden of gastroparesis in the United States, 2006 to 2013. J Clin Gastroenterol 2019;53:109-113.

7. Wang YR, Fisher RS, Parkman HP. Gastroparesis-related hospitalizations in the United States: trends, characteristics, and outcomes, 19952004. Am J Gastroenterol 2008;103:313-322.

8. Lacy BE, Crowell MD, Mathis C, Bauer D, Heinberg LJ. Gastroparesis: quality of life and health care utilization. J Clin Gastroenterol 2018;52:20-24.

9. Agency for Healthcare Research and Quality. Healthcare cost and utilization project 2014. Available from URL: https://www.hcup-us.ahrq. gov/nrdoverview.jsp (accessed 4 June, 2021).

10. Parkman HP, Yates K, Hasler WL, et al. Cholecystectomy and clinical presentations of gastroparesis. Dig Dis Sci 2013;58:1062-1073.

11. Eagon JC, Miedema BW, Kelly KA. Postgastrectomy syndromes. Surg Clin North Am 1992;72:445-465.

12. Elixhauser A, Steiner C, Harris DR, Coffey RM. Comorbidity measures for use with administrative data. Med Care 1998;36:8-27.

13. Parkman HP, Camilleri M, Farrugia G, et al. Gastroparesis and functional dyspepsia: excerpts from the AGA/ANMS meeting. Neurogastroenterol Motil 2010;22:113-133.

14. Uppalapati SS, Ramzan Z, Fisher RS, Parkman HP. Factors contributing to hospitalization for gastroparesis exacerbations. Dig Dis Sci 2009;54:2404-2409.

15. Albers JW, Herman WH, Pop-Busui R, et al. Effect of prior intensive insulin treatment during the diabetes control and complications trial (DCCT) on peripheral neuropathy in type 1 diabetes during the epidemiology of diabetes interventions and complications (EDIC) study. Diabetes Care 2010;33:1090-1096.

16. Callaghan BC, Little AA, Feldman EL, Hughes RA. Enhanced glucose control for preventing and treating diabetic neuropathy. Cochrane Database Syst Rev 2012;6:CD007543.

17. Pop-Busui R, Low PA, Waberski BH, et al. Effects of prior intensive insulin therapy on cardiac autonomic nervous system function in type 1 diabetes mellitus: the diabetes control and complications trial/epidemiology of diabetes interventions and complications study (DCCT/EDIC). Circulation 2009;119:2886-2893.

18. Hyett B, Martinez FJ, Gill BM, et al. Delayed radionucleotide gastric emptying studies predict morbidity in diabetics with symptoms of gastroparesis. Gastroenterology 2009;137:445-452.

19. Parkman HP, Yates K, Hasler WL, et al. Clinical features of idiopathic gastroparesis vary with sex, body mass, symptom onset, delay in gastric emptying, and gastroparesis severity. Gastroenterology 2011;140:101115.

20. Shafi MA, Pasricha PJ. Post-surgical and obstructive gastroparesis. Curr Gastroenterol Rep 2007;9:280-285.

21. Cherian D, Sachdeva P, Fisher RS, Parkman HP. Abdominal pain is a frequent symptom of gastroparesis. Clin Gastroenterol Hepatol 2010;8:676-681. 
22. Tack J, Sarnelli G. Serotonergic modulation of visceral sensation: upper gastrointestinal tract. Gut 2002;51(suppl 1):i77-i80.

23. Parkman HP, Van Natta ML, Abell TL, et al. Effect of nortriptyline on symptoms of idiopathic gastroparesis: the NORIG randomized clinical trial. JAMA 2013;310:2640-2649.

24. Talley NJ, Ford AC. Functional dyspepsia. N Engl J Med 2015;373:1853-1863.

25. Vedula SS, Bero L, Scherer RW, Dickersin K. Outcome reporting in industry-sponsored trials of gabapentin for off-label use. N Engl J Med 2009;361:1963-1971.

26. Kim $\mathrm{CH}$, Nelson DK. Venting percutaneous gastrostomy in the treatment of refractory idiopathic gastroparesis. Gastrointest Endosc 1998;47:67-70.

27. Murr MM, Sarr MG, Camilleri M. The surgeon's role in the treatment of chronic intestinal pseudoobstruction. Am J Gastroenterol 1995;90:2147-2151.

28. Pitt HA, Mann LL, Berquist WE, Ament ME, Fonkalsrud EW, DenBesten L. Chronic intestinal pseudo-obstruction. Management with total parenteral nutrition and a venting enterostomy. Arch Surg 1985;120:614618.

29. Howard L, Ashley C. Management of complications in patients receiving home parenteral nutrition. Gastroenterology 2003;124:1651-1661.

30. Buchman AL. Complications of long-term home total parenteral nutrition: their identification, prevention and treatment. Dig Dis Sci 2001;46:1-18.
31. Diamanti A, Gambarara M, Knafelz D, et al. Prevalence of liver complications in pediatric patients on home parenteral nutrition: indications for intestinal or combined liver-intestinal transplantation. Transplant Proc 2003;35:3047-3049.

32. Toro JP, Lytle NW, Patel AD, et al. Efficacy of laparoscopic pyloroplasty for the treatment of gastroparesis. J Am Coll Surg 2014;218:652-660.

33. Shada AL, Dunst CM, Pescarus R, et al. Laparoscopic pyloroplasty is a safe and effective first-line surgical therapy for refractory gastroparesis. Surg Endosc 2016;30:1326-1332.

34. Mancini SA, Angelo JL, Peckler Z, Philp FH, Farah KF. Pyloroplasty for refractory gastroparesis. Am Surg 2015;81:738-746.

35. Zihni AM, Dunst CM, Swanström LL. Surgical management for gastroparesis. Gastrointest Endosc Clin N Am 2019;29:85-95.

36. Jehangir A, Parkman HP. Cannabinoid use in patients with gastroparesis and related disorders: prevalence and benefit. Am J Gastroenterol 2019;114:945-953.

37. McCallum RW, Bashashati M. Cannabis for gastroparesis: hype or hope? Am J Gastroenterol 2019;114:865-866.

38. Shahsavari D, Zhao H, Ehrlich AC, et al. Factors associated with hospital admissions and readmissions in patients with gastroparesis using the nationwide readmission database. J Clin Gastroenterol 2020;54:801-805.

39. Qayed E, Muftah M. Frequency of hospital readmission and care fragmentation in gastroparesis: a nationwide analysis. World J Gastrointest Endosc 2018;10:200-209. 